\title{
Flexible and Stretchable Liquid Metal Electrodes Working at Sub-Zero Temperature and Their Applications
}

\author{
Peng Xiao ${ }^{1}(\mathrm{D})$, Ju-Hyung $\mathrm{Kim}^{2,3, *(\mathbb{D})}$ and Soonmin Seo ${ }^{1, *(\mathbb{D})}$ \\ 1 Department of Bionano Technology, Gachon University, Seongnam 13120, Korea; zhongpengxiao@gmail.com \\ 2 Department of Chemical Engineering, Ajou University, Suwon 16499, Korea \\ 3 Department of Energy Systems Research, Ajou University, Suwon 16499, Korea \\ * Correspondence: juhyungkim@ajou.ac.kr (J.-H.K.); soonmseo@gachon.ac.kr (S.S.)
}

Citation: Xiao, P.; Kim, J.-H.; Seo, S. Flexible and Stretchable Liquid Metal Electrodes Working at Sub-Zero Temperature and Their Applications. Materials 2021, 14, 4313. https:// doi.org/10.3390/ma14154313

Academic Editor: Ricardo Alcántara

Received: 18 June 2021

Accepted: 30 July 2021

Published: 2 August 2021

Publisher's Note: MDPI stays neutral with regard to jurisdictional claims in published maps and institutional affiliations.

Copyright: (c) 2021 by the authors. Licensee MDPI, Basel, Switzerland. This article is an open access article distributed under the terms and conditions of the Creative Commons Attribution (CC BY) license (https:/ / creativecommons.org/licenses/by/ $4.0 /)$.

\begin{abstract}
We investigated characteristics of highly flexible and stretchable electrodes consisting of Galinstan (i.e., a gallium-based liquid metal alloy) under various conditions including sub-zero temperature (i.e., $<0^{\circ} \mathrm{C}$ ) and demonstrated solar-blind photodetection via the spontaneous oxidation of Galinstan. For this work, a simple and rapid method was introduced to fabricate the Galinstan electrodes with precise patterns and to exfoliate their surface oxide layers. Thin conductive films possessing flexibility and stretchability can be easily prepared on flexible substrates with large areas through compression of a dried suspension of Galinstan microdroplets. Furthermore, a laser marking machine was employed to facilitate patterning of the Galinstan films at a high resolution of $20 \mu \mathrm{m}$. The patterned Galinstan films were used as flexible and stretchable electrodes. The electrical conductivity of these electrodes was measured to be $\sim 1.3 \times 10^{6} \mathrm{~S} \mathrm{~m}^{-1}$, which were still electrically conductive even if the stretching ratio increased up to $130 \%$ below $0{ }^{\circ} \mathrm{C}$. In addition, the surface oxide (i.e., $\mathrm{Ga}_{2} \mathrm{O}_{3}$ ) layers possessing photo-responsive properties were spontaneously formed on the Galinstan surfaces under ambient conditions, which could be solely exfoliated using elastomeric stamps. By combining Galinstan and its surface oxide layers, solar-blind photodetectors were successfully fabricated on flexible substrates, exhibiting a distinct increase of up to $14.7 \%$ in output current under deep ultraviolet irradiation ( $254 \mathrm{~nm}$ wavelength) with an extremely low light intensity of $0.1 \mathrm{~mW} \mathrm{~cm}^{-2}$, whereas no significant change was observed under visible light irradiation.
\end{abstract}

Keywords: liquid metals; gallium alloys; Galinstan; flexible electronics photodetectors; solarblind photodetection

\section{Introduction}

Recently, liquid metals based on gallium (Ga) alloys have received increasing attention, owing to their outstanding electrical and mechanical properties [1-3]. The Ga-based metal alloys that exist as virtually non-toxic liquids at room temperature show not only excellent stretchability and deformability but also environmental friendliness and recyclability. In this context, extensive research efforts have been devoted to the development of various applications using Ga-based metal alloys, such as sensors [4], reconfigurable antennas [5], and soft electrodes [6]. These fluidic metal alloys also show great potential for electronic skins [7,8] and wearable electronics [9-11]. Among various Ga-based metal alloys, Galinstan $(68.5 \% \mathrm{Ga}, 21.5 \%$ indium (In), and 10\% tin (Sn)) has been notably studied in recent years due to its remarkably low toxicity and melting point $\left(\sim-19^{\circ} \mathrm{C}\right)$ [12], which is also suitable for flexible and stretchable devices operating below $0{ }^{\circ} \mathrm{C}$ compared to other eutectic gallium indium (EGaIn).

Although Galinstan shows outstanding properties including flexibility and stretchability even under cold conditions, its high surface tension and rapid oxidation rate hinder the fabrication of desirable patterns for electronic devices and circuits in comparison with other functional materials [13-15]. Various methods for Galinstan patterning thus have been developed and enhanced, including microfluidic injection [16-21], photolithography [22,23], 
stencil lithography [24-27], imprint lithography [28], microcontact printing [29,30], and composite material synthesis [31]. Each of these methods has individual advantages (i.e., high processability, high resolution limits, high stability, or cost-effective fabrication); however, integrating all the advantageous elements is still a challenge. For instance, the demanding process conditions for delaying surface oxidation or preventing leakage of the liquid metal or the low patterning resolution limits still need to be improved depending on the method. Laser ablation is one of the patterning methods with high processability, enabling the rapid fabrication of electrodes with complex features [32,33]. Although the pattern resolution is limited by the beam spot of laser, which is normally in the range of tens to hundreds of micrometers, this method can be directly employed for various applications without laborious pre- and/or post-treatments.

In addition, it is worth noting that the spontaneous oxidation of Galinstan in air leads to the formation of thin $\mathrm{Ga}$ oxide $\left(\mathrm{Ga}_{2} \mathrm{O}_{3}\right)$ films on Galinstan surfaces $[3,34,35]$. $\mathrm{Ga}_{2} \mathrm{O}_{3}$ with a wide bandgap $(\sim 4.9 \mathrm{eV})$, which is rapidly formed on Galinstan surfaces in less than one second during the patterning processes in air [2], is transparent in the visible light region and exhibits high light-absorption coefficients in the deep ultraviolet (UV) region [35-38]. The surface oxide layers normally degrade the metallic properties of Galinstan; however, these layers are also expected to be utilized for solar-blind photodetection (i.e., deep UV detection insensitive to solar radiation) if they can be neatly separated from the bulk material [39].

Herein, we investigated characteristics of the Galinstan electrodes to verify flexibility and stretchability under various conditions including sub-zero temperature (i.e., $<0{ }^{\circ} \mathrm{C}$ ). For this study, a simple and rapid method was employed to fabricate the Galinstan electrodes with precise patterns. Thin Galinstan films with high electrical conductivity were uniformly deposited on flexible polydimethylsiloxane (PDMS) substrates by the compression of Galinstan microdroplets and sequentially patterned using a fiber laser marking machine. The transparent PDMS substrates were found to be undamaged by a laser with a wavelength of $1064 \mathrm{~nm}$, and only the Galinstan layers were ablated according to the designed electrode shapes. In addition, the surface oxide (i.e., $\mathrm{Ga}_{2} \mathrm{O}_{3}$ ) layers of the Galinstan electrodes were also examined to confirm their potential for solar-blind photodetection. For the photoactive components, the thin $\mathrm{Ga}_{2} \mathrm{O}_{3}$ films, spontaneously formed on the Galinstan surfaces, were exfoliated using elastomeric PDMS stamps [39,40] and then transferred onto the patterned Galinstan electrodes to complete the device structure for solar-blind photodetection. By combining Galinstan and $\mathrm{Ga}_{2} \mathrm{O}_{3}$ films, sensitive solar-blind photodetectors were successfully fabricated on flexible substrates. The photodetectors showed a distinct increase of up to $\sim 15.1 \%$ in output current under deep UV irradiation (254 nm wavelength) with an extremely low light intensity of $0.1 \mathrm{~mW} \mathrm{~cm}^{-2}$, whereas no significant change was observed under visible light irradiation.

\section{Materials and Methods}

\subsection{Preparation of Galinstan Microdroplets}

Galinstan (68.5 wt.\% Ga, $21.5 \mathrm{wt} . \% \mathrm{In}$, and $10.0 \mathrm{wt} . \% \mathrm{Sn}$ ) and PDMS (SYLGARD 184) were purchased from Geratherm Medical AG (Geratal, Germany) and DOW (Midland, MI, USA), respectively. EGaIn (75.5 wt.\% Ga and $24.5 \mathrm{wt} . \% \mathrm{In})$ was purchased from Sigma-Aldrich Korea (Seoul, Korea). To prepare the Galinstan microdroplets, $0.5 \mathrm{~g}$ of Galinstan was sonicated in ethanol for $30 \mathrm{~min}(80 \mathrm{~W}, 40 \mathrm{KHz})$. Galinstan was well dispersed during the sonication process and rapidly stabilized by surface oxidation in ethanol, resulting in the suspension of Galinstan microdroplets $(<10 \mu \mathrm{m})$ as shown in Figure 1a. The same procedure was repeatedly performed for the preparation of the EGaIn microdroplets. 
a
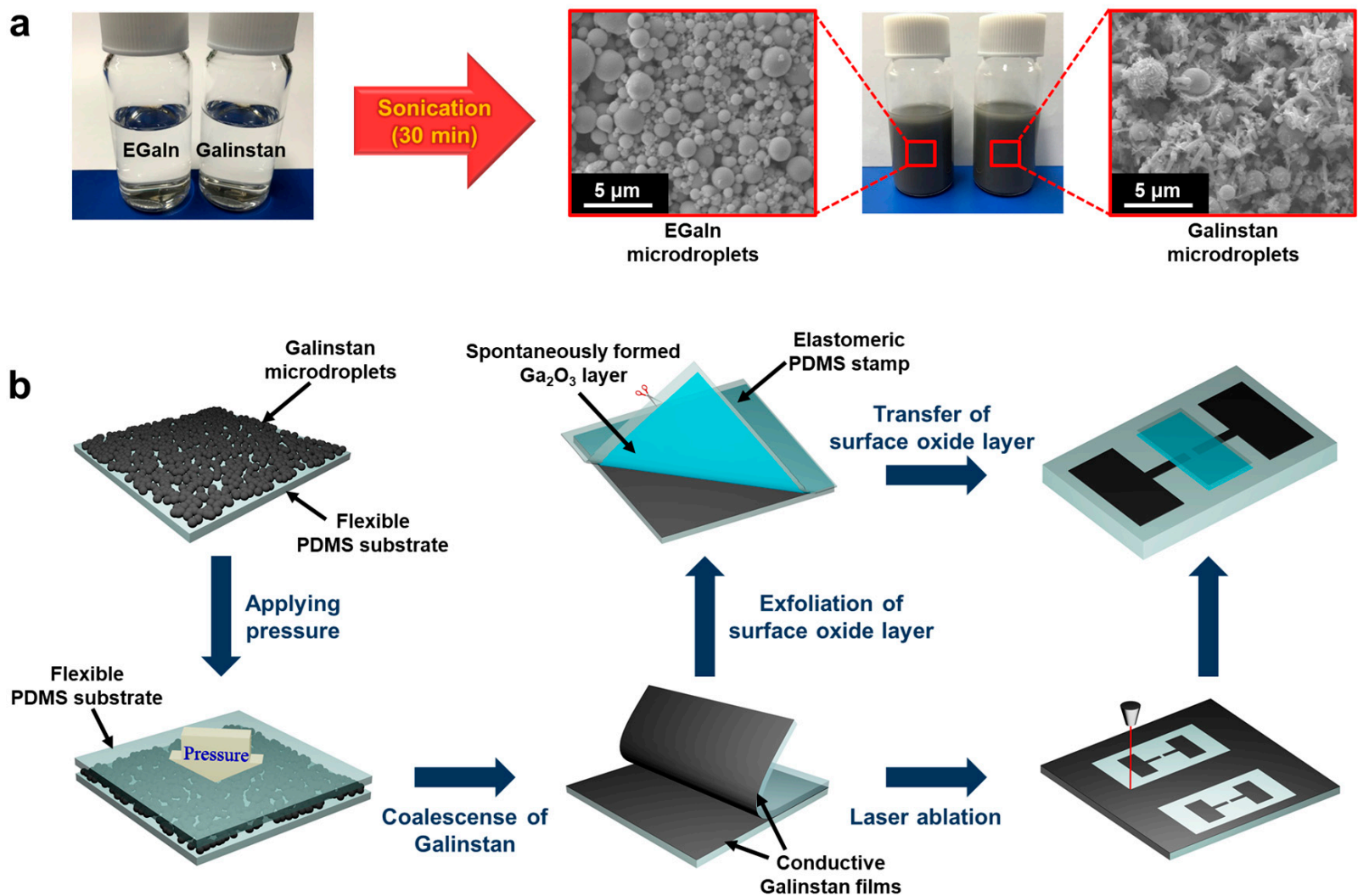

Figure 1. (a) Preparation of suspensions comprising EGaIn and Galinstan microdroplets by sonication. (b) Schematic illustration of the fabrication of flexible solar-blind photodetector using Galinstan microdroplets.

\subsection{Preparation of Elastomeric PDMS Substrates and Stamps}

For the PDMS substrates, the PDMS precursor, consisting of a silicone elastomer base and a curing agent (in a 10:1 weight ratio), was poured onto a flat Petri dish, and subsequently degassed in a vacuum desiccator for $1 \mathrm{~h}$. The sample was cured at $80^{\circ} \mathrm{C}$ for $1 \mathrm{~h}$ in a convection oven. After thermal curing, the PDMS film was easily peeled off from the Petri dish and then cut into $50 \mathrm{~mm} \times 50 \mathrm{~mm}$ specimens. The thickness of each substrate was measured as $\sim 1 \mathrm{~mm}$. Sticky elastomeric PDMS stamps were individually prepared to exfoliate the thin $\mathrm{Ga}_{2} \mathrm{O}_{3}$ films. The mixing ratio of the PDMS precursor was modified to a 11:1 weight ratio to delay the saturation of cross-linking and enhance its adhesive properties, and the same preparation procedure as for the PDMS substrates was followed.

\subsection{Fabrication of Patterned Galinstan Electrodes}

The suspension containing the Galinstan microdroplets was drop-dispensed onto the flat PDMS substrate and then slowly dried at $30{ }^{\circ} \mathrm{C}$ for $24 \mathrm{~h}$ to avoid the formation of structural defects induced by rapid evaporation of the solvent. The dried suspension of the microdroplets in thin-film form was covered with another flat PDMS substrate and subsequently pressed at a pressure of $15 \mathrm{MPa}$ for $5 \mathrm{~s}$ to collapse the surface oxide layers and connect Galinstan. After releasing the pressure, the upper PDMS mold was peeled off from the bottom PDMS substrate, resulting in the formation of thin conductive Galinstan films deposited on both PDMS substrates.

The Galinstan films were patterned using a laser marking machine (50 W, Dongil Laser Technology, Gwangju, Korea). The scanning speed of the laser marking machine was $600 \mathrm{~mm} \mathrm{~s}^{-1}$, and the power intensity was $1.0 \%$ of its maximum power (i.e., $0.5 \mathrm{~W}$ ). A high resolution of $20 \mu \mathrm{m}$ was achieved in the Galinstan patterning process by this laser ablation method. 


\subsection{Characterization of Flexible and Stretchable Liquid Metal Electrodes}

To investigate characteristics of the liquid metal electrodes under various conditions, thin conductive films $\left(15 \times 25 \mathrm{~mm}^{2}\right)$ of Galinstan and EGaIn were individually prepared with the same procedure. The thickness of each film was $1 \mu \mathrm{m}$. A semiconductor characterization system (4200-SCS, Keithley, Beaverton, OR, USA) was used for the measurements.

\subsection{Fabrication of Flexible Solar-Blind Photodetectors Using $\mathrm{Ga}_{2} \mathrm{O}_{3}$}

The elastomeric PDMS stamp was brought into contact with the surface of the Galinstan film. A thin $\mathrm{Ga}_{2} \mathrm{O}_{3}$ film $(<10 \mathrm{~nm})$, which was spontaneously formed on the Galinstan surface, was attached to the sticky PDMS stamp and easily exfoliated from the Galinstan film by peeling off the stamp. The transparent $\mathrm{Ga}_{2} \mathrm{O}_{3}$ film on the PDMS stamp was cut and placed between two patterned Galinstan electrodes to complete the device structure.

\subsection{Measurements}

A semiconductor parameter analyzer (Keithley 4200, Beaverton, OR, USA) and resistivity meter (Loresta-GX MCP-T700, Mitsubishi Chemical Analytech, Yamato, Japan) were used to measure electrical properties and perform bending tests on the devices. Photocurrent measurements were performed for deep UV and visible regions using a UV lamp (8 W, Vilber Lourmat, Collégien, France) and a halogen lamp (FOK-100W, Fiber Optic Korea, Cheonan, Korea). The surface morphology was also investigated using atomic force microscopy (AFM; Nanoscope IIIa, Digital Instruments, Bresso, Italy) and scanning electron microscopy (SEM; JSM-7500F, Tokyo, Japan).

\section{Results and Discussion}

Suspensions comprising microdroplets of Galinstan and EGaIn were individually prepared as shown in Figure 1a. It exhibited a matt dark gray color due to diffuse reflections and surface oxide layers of the microdroplets. As schematically illustrated in Figure 1b, the suspension was drop-dispensed onto a flat PDMS substrate and then slowly dried at $30{ }^{\circ} \mathrm{C}$ for $24 \mathrm{~h}$. As the slow drying process hindered the formation of structural defects induced by rapid evaporation of the solvent, the microdroplets were densely aggregated in thin-film form with high uniformity. For Galinstan, the size of each microdroplet was less than $5 \mu \mathrm{m}$, and rod-shaped particles were also observed between the rounded droplets. It is well known that rounded Galinstan microdroplets are surrounded by a thin layer of carbon and $\mathrm{Ga}_{2} \mathrm{O}_{3}$, of which the inner core is composed of $\mathrm{Ga}$, In, and Sn $[41,42]$. As previously reported, the rod-shaped particles possibly consisted of Ga oxide monohydroxide $((\mathrm{GaO}) \mathrm{OH})$ [43]. Note that $\mathrm{Ga}$ in Ga-based metal alloys can react with decomposed $\mathrm{OH}^{-}$in the presence of $\mathrm{O}_{2}$, leading to the crystallization of $(\mathrm{GaO}) \mathrm{OH}$ as follows:

$$
2 \mathrm{Ga}+2 \mathrm{OH}^{-}+\mathrm{O}_{2} \rightarrow 2(\mathrm{GaO}) \mathrm{OH}
$$

The amount of rod-shaped particles is significantly less than that of round particles, and it is expected to be further reduced at low-temperature conditions because the crystallization strongly depends on heat and reactive oxygen species originating from sonication [44,45].

The thin film comprising aggregated Galinstan microdroplets was not electrically conductive because each droplet was fully covered by a non-conductive $\mathrm{Ga}_{2} \mathrm{O}_{3}$ layer. Thus, another flat PDMS substrate for protecting Galinstan was brought into contact with the thin film, and an external pressure of $15 \mathrm{MPa}$ was sequentially applied to the sample to collapse the surface $\mathrm{Ga}_{2} \mathrm{O}_{3}$ layers and connect Galinstan. With the collapse of the surface $\mathrm{Ga}_{2} \mathrm{O}_{3}$ layers, the Galinstan microdroplets were connected to achieve a continuous phase between the two PDMS substrates. After peeling off the upper PDMS substrate from the bottom PDMS substrate, thin Galinstan films were consequently formed on both PDMS substrates (see Figure 2a). The thickness of each glossy film was measured to be less than $1 \mu \mathrm{m}$, of which the surface partially cracked due to rapid surface oxidation during the peeling process. 
a

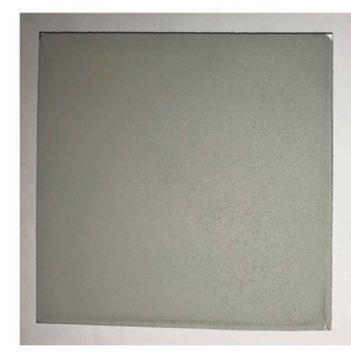

C

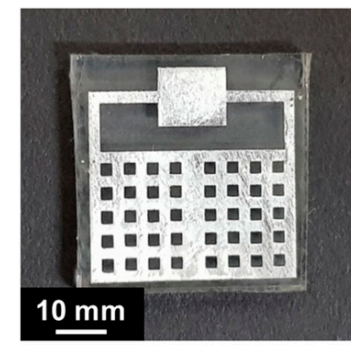

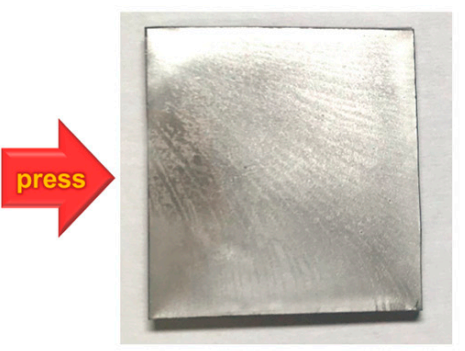

b
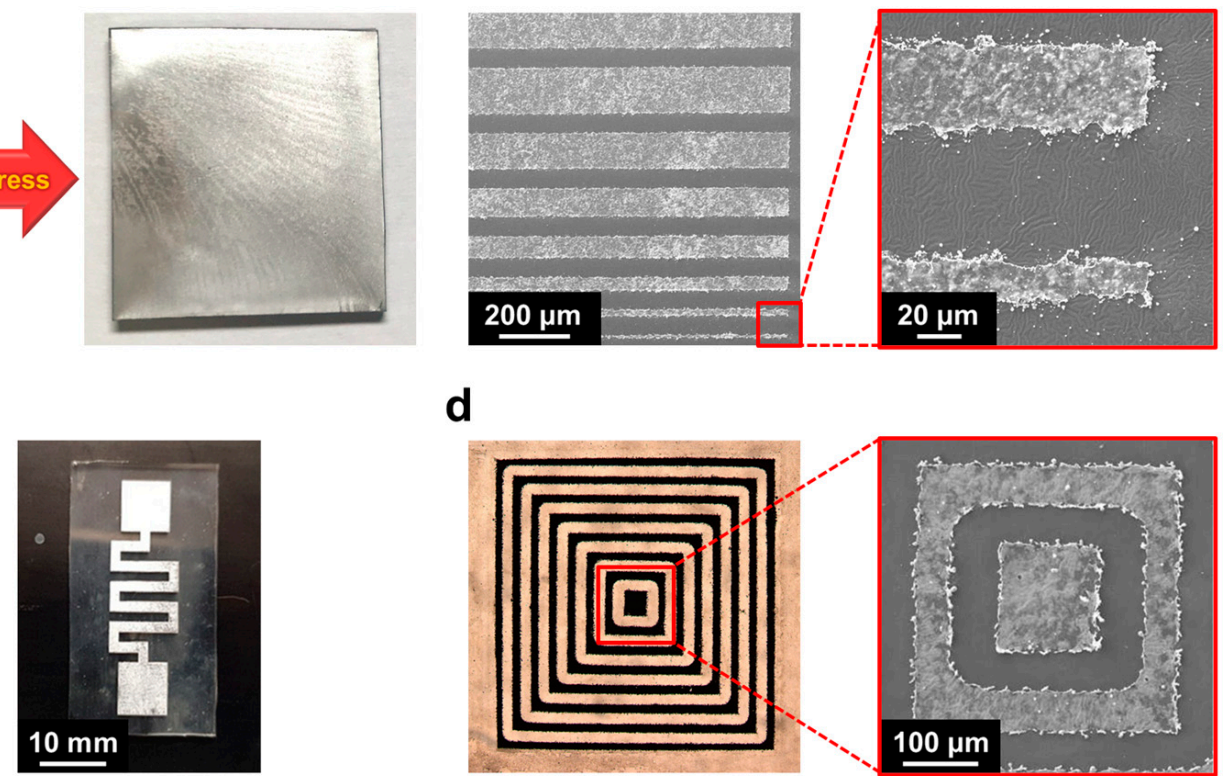

d

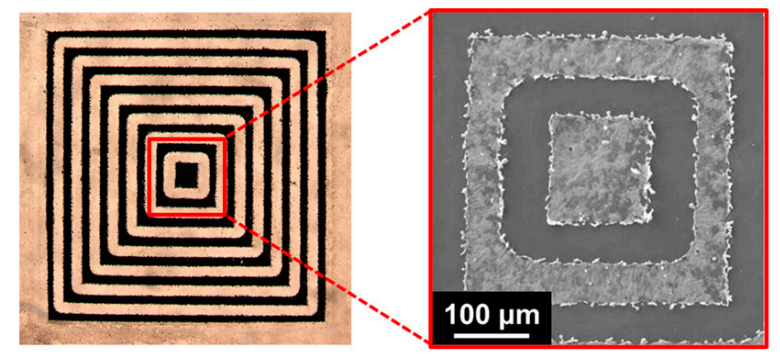

Figure 2. (a) Photographs of (left) a dried suspension of Galinstan microdroplets in thin-film form on a flexible PDMS substrate, and (right) a conductive Galinstan film fabricated by compression and separation using PDMS. (b) SEM images of patterned Galinstan films, of which the minimum line width is $\sim 20 \mu \mathrm{m}$. (c) Photographs of exemplary Galinstan films, patterned by laser ablation. (d) Optical microscopy image and its close-up SEM image of an exemplary Galinstan structure with complex pattern.

A fiber laser marking machine $(\lambda \sim 1064 \mathrm{~nm})$ was employed for the direct patterning of the Galinstan films, enabling the fabrication of accurate and desirable features with sub-100 $\mu \mathrm{m}$ resolution. Various exemplary features of the patterned Galinstan films are shown in Figure $2 b-d$. The smallest feature size of the Galinstan line was $20 \mu \mathrm{m}$. Laser ablation is a fast and precise method for patterning liquid metal electrodes, facilitating the fabrication of complex and hollow patterns. In addition, this light-based patterning method does not cause significant damage to the transparent substrates, such as glass and PDMS, which do not directly absorb the energy from a fiber laser. After completing the laser ablation process, partial buckling was observed on the PDMS substrates.

The electrical resistance and conductivity of the Galinstan film were measured corresponding to the structural deformation of the PDMS substrate. For the measurements of electrical properties, the patterned Galinstan electrode $(80 \mu \mathrm{m} \times 5 \mathrm{~mm})$ was used as shown in Figure 3. Its thickness was less than $1 \mu \mathrm{m}$. The electrical resistance and conductivity were initially measured as $48.3 \Omega$ and $\sim 1.3 \times 10^{6} \mathrm{~S} \mathrm{~m}^{-1}$, respectively. In response to the deformation ratio, the electrical resistance gradually increased to $73.8 \Omega$, corresponding to an electrical conductivity of $\sim 8.5 \times 10^{5} \mathrm{~S} \mathrm{~m}^{-1}$. In comparison with pure Galinstan, in which the electrical conductivity was found to be $\sim 3.5 \times 10^{6} \mathrm{~S} \mathrm{~m}^{-1}$, the relatively low electrical conductivity of the Galinstan films used in this work could be attributed to the partial cracks and insulating components remaining in the films, such as $(\mathrm{GaO}) \mathrm{OH}$ and $\mathrm{Ga}_{2} \mathrm{O}_{3}$. However, since the majority of the film components were Galinstan, the fabricated films still possessed electrical conductivity high enough to be used as flexible electrodes. It should be noted that the electrical conductivity of the fabricated Galinstan film $\left(\sim 1.3 \times 10^{6} \mathrm{~S} \mathrm{~m}^{-1}\right)$ is slightly lower than that of the thin EGaIn film $\left(\sim 2.2 \times 10^{6} \mathrm{~S} \mathrm{~m}^{-1}\right)$ prepared using the same procedure [39]. It is possibly originating from the content of the insulating material in the suspension. As shown in Figure 1a, the content of the rod-shaped particles in the Galinstan suspension is significantly higher than in the EGaIn suspension under our experimental conditions, which may cause a decrease in overall electrical conductivity. 

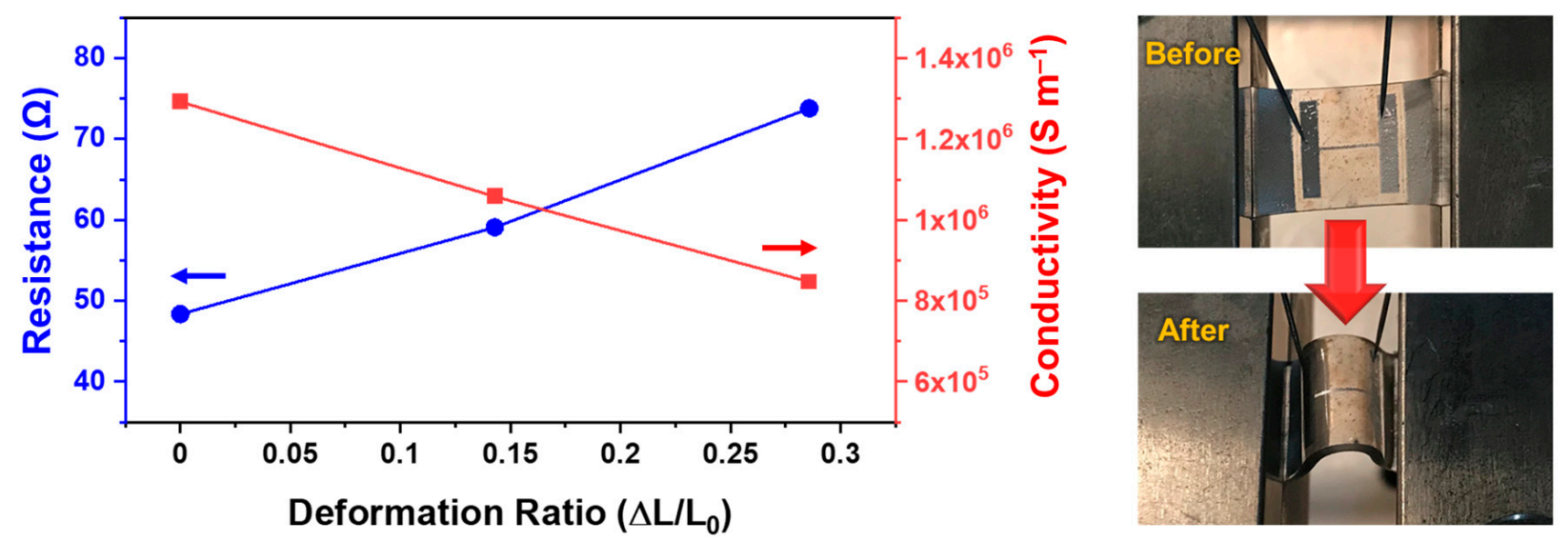

Figure 3. Electrical resistance and conductivity of a line-patterned Galinstan electrode $(80 \mu \mathrm{m} \times 5 \mathrm{~mm})$ corresponding to the structural deformation of the PDMS substrate.

One advantageous property of Galinstan is the liquid phase, maintaining its flexibility and stretchability, even below $0{ }^{\circ} \mathrm{C}$. To compare with EGaIn, electrical resistances of the two materials were measured corresponding to lateral stretching (up to $\sim 130 \%$ ) of the PDMS substrates. For the measurements, the flat Galinstan and EGaIn electrodes were individually prepared on the PDMS substrates $\left(15 \times 25 \times 1 \mathrm{~mm}^{3}\right)$ and then stretched up to $130 \%$ at room temperature and $-10{ }^{\circ} \mathrm{C}$, respectively. Changes in the electrical resistances upon lateral stretching are shown in Figure 4a. At room temperature, the electrical resistances of both materials slightly increased with the stretching ratios, which is possibly originating from structural deformation [26]. At the temperature of $-10^{\circ} \mathrm{C}$, the cracks were generated inside the EGaIn film upon the lateral stretches (see Figure $4 \mathrm{~b}$ ), leading to significant reduction in the film continuity. When the stretching ratio was above $110 \%$, the electrical conductivity of the EGaIn film was thus not observed. However, differently from EGaIn, the Galinstan film was still electrically conductive even if the stretching ratio increased up to $130 \%$. These results were caused by the difference in the melting points of the two materials (i.e., $\sim-19{ }^{\circ} \mathrm{C}$ for Galinstan and $\sim 16{ }^{\circ} \mathrm{C}$ for EGaIn). At $-10{ }^{\circ} \mathrm{C}$, the EGaIn film in the solid phase was significantly damaged, whereas the Galinstan film in the liquid phase showed excellent film continuity (see Figure 4c).

a

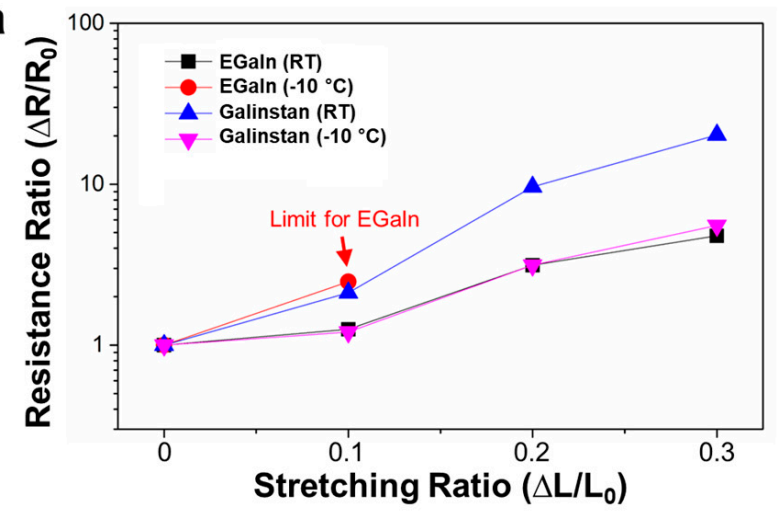

b

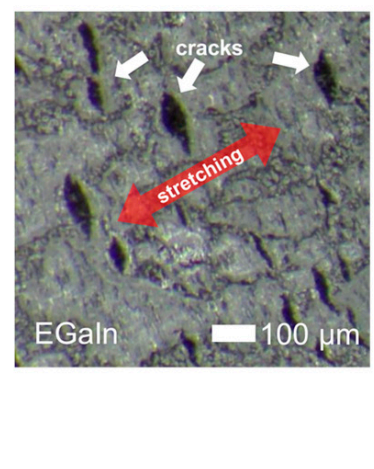

C

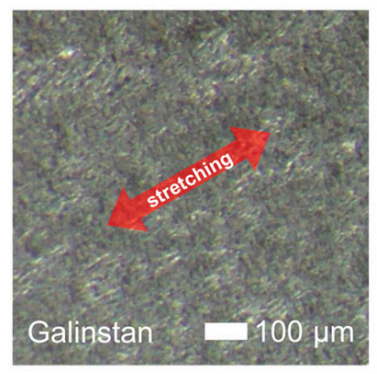

Figure 4. (a) Changes in electrical resistances of EGaIn and Galinstan films corresponding to lateral stretching at varied temperatures (i.e., room temperature (RT) and $-10^{\circ} \mathrm{C}$ ). (b) SEM image of a cracked EGaIn film after lateral stretching below $0{ }^{\circ} \mathrm{C}$. (c) SEM image of a stretched Galinstan film below $0{ }^{\circ} \mathrm{C}$, exhibiting excellent film continuity.

In addition, the $\mathrm{Ga}_{2} \mathrm{O}_{3}$ layer, which was spontaneously formed on the Galinstan film, was neatly exfoliated using an elastomeric PDMS stamp for further investigation. It should be noted that inherently high adhesion between the thin oxide shell and PDMS was reported [42], and the elastomeric PDMS stamps enabled intimate contact with the oxide 
surfaces. In this work, the mixing ratio of the PDMS precursor was further modified to enhance the adhesive properties, demonstrating excellent contact characteristics with a relatively rough $\mathrm{Ga}_{2} \mathrm{O}_{3}$ surface. The elastomeric PDMS stamp was placed on the surface of the Galinstan film without applying any external pressure and was then detached. In this process, the transparent $\mathrm{Ga}_{2} \mathrm{O}_{3}$ layer was successfully transferred onto the stamp. The transferred $\mathrm{Ga}_{2} \mathrm{O}_{3}$ film was slightly darker than the bare PDMS substrate because small Galinstan residues in the form of islands $(<10 \mu \mathrm{m})$ remained on the substrate, as shown in Figure 5c. However, all Galinstan residues were entirely isolated from each other and wrapped with $\mathrm{Ga}_{2} \mathrm{O}_{3}$, resulting in the formation of a non-metallic film. The thickness of the exfoliated $\mathrm{Ga}_{2} \mathrm{O}_{3}$ film was measured as $\sim 13 \mathrm{~nm}$ using AFM, as shown in Figure $5 \mathrm{~b}$. It is worth noting that the measured thickness of the $\mathrm{Ga}_{2} \mathrm{O}_{3}$ film in this work is thicker than that of the single surface oxide layer of Galinstan (i.e., $\sim 3 \mathrm{~nm}$ ) due to further oxidation during the exfoliation process. The surface roughness and embossed features of the fabricated Galinstan film, as shown in Figure 2d, could also affect the thickness of the $\mathrm{Ga}_{2} \mathrm{O}_{3}$ film.
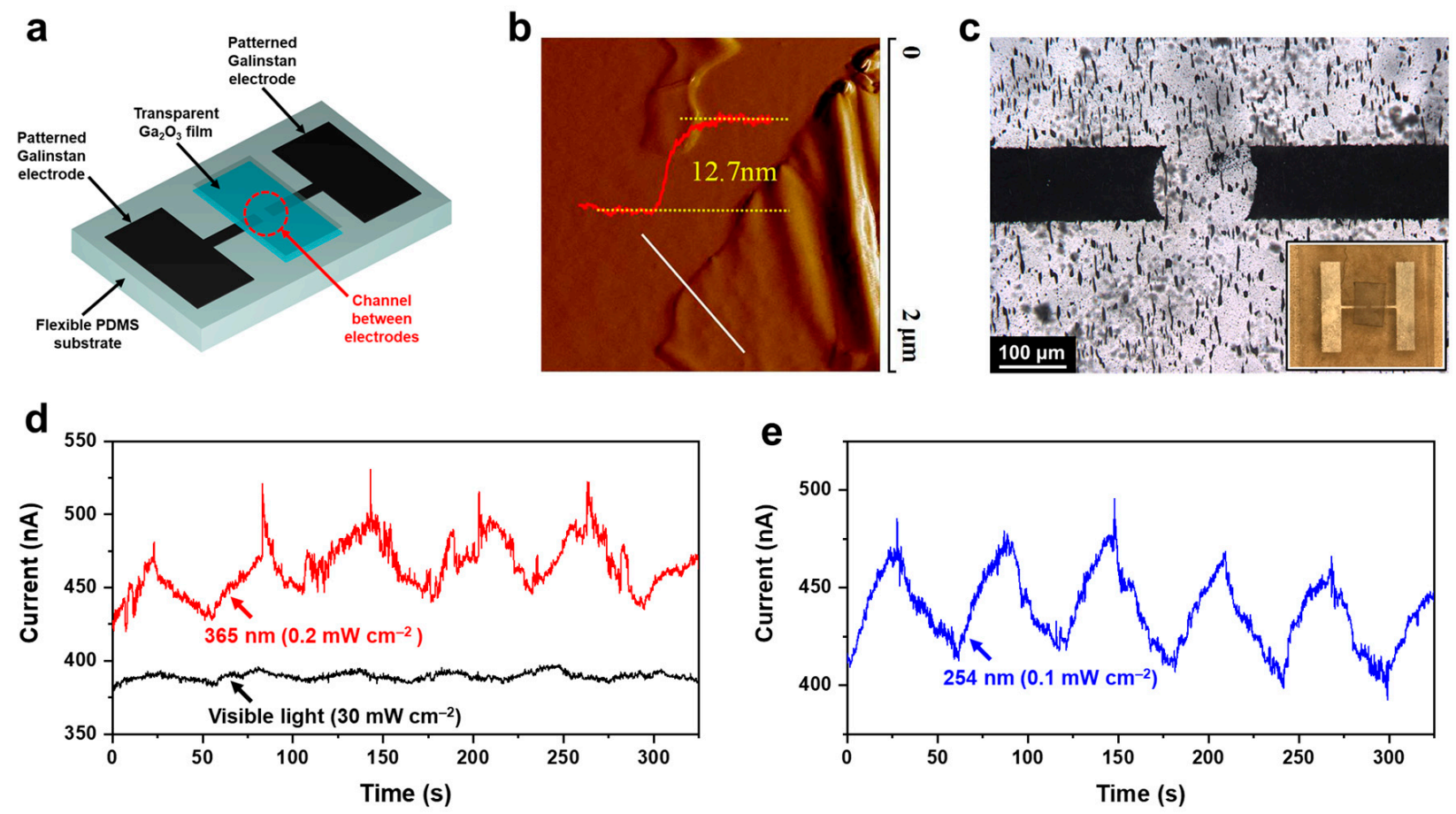

Figure 5. (a) Schematic illustration of solar-blind photodetector. (b) AFM image of transparent $\mathrm{Ga}_{2} \mathrm{O}_{3}$ film, exfoliated using a PDMS stamp. The height profile along the white line is also indicated. (c) Optical microscopy image of a channel between two patterned Galinstan electrodes. The black areas correspond to Galinstan beneath the transparent $\mathrm{Ga}_{2} \mathrm{O}_{3}$ film. Photograph of the fabricated photodetector is also shown in the inset. (d) Output characteristics of the solar-blind photodetector under irradiations of visible light (with a halogen lamp; ranging from 350 to $900 \mathrm{~nm}$ ) and UV light of $365 \mathrm{~nm}$ wavelength, respectively. (e) Output characteristics of the solar-blind photodetector under deep UV irradiation (254 nm wavelength). The on/off switching of each irradiation was manually performed at $30 \mathrm{~s}$ intervals, and the output characteristics were constantly measured at a sample bias voltage of $0.1 \mathrm{~V}$.

To investigate the solar-blind photodetective properties of the exfoliated $\mathrm{Ga}_{2} \mathrm{O}_{3}$ film in consideration of its wide bandgap ( 4.9 eV) [35-38], a channel between two patterned Galinstan electrodes was bridged using $\mathrm{Ga}_{2} \mathrm{O}_{3}$, as shown in Figure 5a. For this work, the conductive Galinstan film, prepared on a large area $(50 \mathrm{~mm} \times 50 \mathrm{~mm})$, was patterned by laser ablation to form a channel. The transparent $\mathrm{Ga}_{2} \mathrm{O}_{3}$ film, individually prepared on the PDMS stamp, was placed between the channel to complete the device structure (see Figure $5 b$ ). The output current was constantly measured at a sample bias voltage of $0.1 \mathrm{~V}$. Under visible light irradiation with a halogen lamp (ranging from 350 to $900 \mathrm{~nm}$ ), the output current only increased by $\sim 2.7 \%$ at a high light intensity of $30 \mathrm{~mW} \mathrm{~cm}^{-2}$ (see 
Figure 5c), and no significant change in the output current was observed at lower light intensities. As the contribution of the short-wavelength region in the emission spectrum is not negligible at high intensity, a small increase in the output current could be detected at light intensities above $30 \mathrm{~mW} \mathrm{~cm}^{-2}$. To confirm this speculation, the output current was also measured under irradiation of UV light of a $365 \mathrm{~nm}$ wavelength, which was contained in the emission spectrum of the halogen lamp, and the output current in effect increased by $\sim 14.9 \%$ at a low light intensity of $0.2 \mathrm{~mW} \mathrm{~cm}^{-2}$. Eventually, under deep UV irradiation (254 nm wavelength) with an extremely low light intensity of $0.1 \mathrm{~mW} \mathrm{~cm}{ }^{-2}$, the output current sensitively increased by up to $15.1 \%$ (see Figure $5 d$ ). These results strongly suggest that the combination of Galinstan and its surface oxide layers can be used for sensitive solar-blind photodetectors that possess remarkable advantages, such as low-cost and easy processability under ambient conditions, and flexibility.

\section{Conclusions}

We investigated characteristics of the flexible and stretchable Galinstan electrodes under various conditions including sub-zero temperature (i.e., $<0{ }^{\circ} \mathrm{C}$ ) and successfully demonstrated solar-blind photodetection via the spontaneous oxidation of Galinstan. In this work, a simple and rapid method was introduced for fabricating the flexible and stretchable Galinstan electrodes with precise patterns and exfoliating the surface oxide layers to complete the device structure enabling solar-blind photodetection. A suspension consisting of Galinstan microdroplets was prepared by sonication. Thin Galinstan films with thickness less than $1 \mu \mathrm{m}$ were uniformly deposited on flexible PDMS substrates by compression of the dried suspension of the microdroplets. The Galinstan films, deposited on a large area $(50 \mathrm{~mm} \times 50 \mathrm{~mm})$, were sequentially patterned using a fiber laser marking machine $(\lambda \sim 1064 \mathrm{~nm})$, and accurate and desirable features with a high resolution of $20 \mu \mathrm{m}$ were fabricated. Although the electrical conductivity of the fabricated films was lower than that of pure Galinstan, they still possessed electrical conductivity high enough to be used as flexible and stretchable electrodes even below $0{ }^{\circ} \mathrm{C}$. For the photoactive components, thin $\mathrm{Ga}_{2} \mathrm{O}_{3}$ layers, spontaneously formed on the Galinstan surfaces, were exfoliated using elastomeric PDMS stamps and successfully transferred onto the patterned Galinstan electrodes to complete the device structure for solar-blind photodetection. The solar-blind photodetectors demonstrated a distinct increase of up to $15.1 \%$ in the output current under deep UV irradiation ( $254 \mathrm{~nm}$ wavelength) with an extremely low light intensity of $0.1 \mathrm{~mW} \mathrm{~cm}{ }^{-2}$, whereas no significant change was observed under visible light irradiation. These results strongly suggest that Galinstan can be used for flexible and stretchable electrodes working under extreme conditions, and the combination with its surface oxide layer also shows great potential for sensitive solar-blind photodetectors that possess outstanding advantages, such as low-cost and easy processability under ambient conditions. We anticipate that these results will contribute to the development of flexible and stretchable electronic devices based on liquid metals, which can lead to further application of sensors under extreme conditions.

Author Contributions: P.X.: conceptualization, methodology, formal analysis, investigation, data curation, visualization, and writing-original draft preparation; J.-H.K.: investigation, data curation, visualization, writing—original draft preparation, review and editing, and supervision; S.S.: conceptualization, formal analysis, visualization, writing - review and editing, supervision, and funding acquisition. All authors have read and agreed to the published version of the manuscript.

Funding: This work was supported by the Basic Science Research Program through the National Research Foundation of Korea (NRF-2021R1F1A1047036 and NRF-2018R1D1A1B07041253).

Institutional Review Board Statement: Not applicable.

Informed Consent Statement: Not applicable.

Data Availability Statement: Data sharing is not applicable to this article.

Conflicts of Interest: The authors declare no conflict of interest. 


\section{References}

1. Zavabeti, A.; Ou, J.Z.; Carey, B.J.; Syed, N.; Orrell-Trigg, R.; Mayes, E.L.H.; Xu, C.; Kavehei, O.; O’Mullane, A.P.; Kaner, R.B.; et al. A liquid metal reaction environment for the room-temperature synthesis of atomically thin metal oxides. Science 2017, 358, 332-335. [CrossRef] [PubMed]

2. Daeneke, T.; Khoshmanesh, K.; Mahmood, N.; de Castro, I.A.; Esrafilzadeh, D.; Barrow, S.J.; Dickey, M.; Kalantar-Zadeh, K. Liquid metals: Fundamentals and applications in chemistry. Chem. Soc. Rev. 2018, 47, 4073-4111. [CrossRef]

3. Carey, B.J.; Ou, J.Z.; Clark, R.M.; Berean, K.J.; Zavabeti, A.; Chesman, A.S.; Russo, S.P.; Lau, D.W.; Xu, Z.-Q.; Bao, Q.; et al. Wafer-scale two-dimensional semi-conductors from printed oxide skin of liquid metals. Nat. Commun. 2017, 8, 1-10.

4. Kim, M.; Seo, S. Flexible pressure and touch sensor with liquid metal droplet based on gallium alloys. Mol. Cryst. Liq. Cryst. 2019, 685, 40-46. [CrossRef]

5. Elassy, K.S.; Akau, T.K.; Shiroma, W.A.; Seo, S.; Ohta, A.T. Low-cost rapid fabrication of conformal liquid-metal patterns. Appl. Sci. 2019, 9, 1565. [CrossRef]

6. Yu, Y.; Wang, Q.; Wu, Y.H.; Liu, J. Liquid metal soft electrode triggered discharge plasma in aqueous solution. RSC Adv. 2016, 6, 114773-114778. [CrossRef]

7. Hong, Y.J.; Jeong, H.; Cho, K.W.; Lu, N.; Kim, D. Wearable and implantable devices for cardiovascular healthcare: From monitoring to therapy based on flexible and stretchable electronics. Adv. Funct. Mater. 2019, 29. [CrossRef]

8. Chen, L.Y.; Tee, B.C.-K.; Chortos, A.L.; Schwartz, G.; Tse, V.; Lipomi, D.J.; Wong, H.-S.P.; McConnell, M.V.; Bao, Z. Continuous wireless pressure monitoring and mapping with ultra-small passive sensors for health monitoring and critical care. Nat. Commun. 2014, 5, 5028. [CrossRef] [PubMed]

9. Wang, S.; Xu, J.; Wang, W.; Wang, G.-J.N.; Rastak, R.; Molina-Lopez, F.; Chung, J.W.; Niu, S.; Feig, V.R.; Lopez, J.; et al. Skin electronics from scalable fabrication of an intrinsically stretchable transistor array. Nat. Cell Biol. 2018, 555, 83-88. [CrossRef] [PubMed]

10. Yang, J.C.; Mun, J.; Kwon, S.Y.; Park, S.; Bao, Z.; Park, S. Electronic skin: Recent progress and future prospects for skin-attachable devices for health monitoring, robotics, and prosthetics. Adv. Mater. 2019, 31, 1904765. [CrossRef]

11. Yang, Y.; Han, J.; Huang, J.; Sun, J.; Wang, Z.L.; Seo, S.; Sun, Q. Stretchable energy-harvesting tactile interactive interface with liquid-metal-nanoparticle-based electrodes. Adv. Funct. Mater. 2020, 30, 1909652. [CrossRef]

12. Liu, T.; Sen, P.; Kim, C.-J. Characterization of nontoxic liquid-metal alloy galinstan for applications in microdevices. J. Microelectromechanical Syst. 2011, 21,443-450. [CrossRef]

13. Shin, J.; Jeong, B.; Kim, J.; Nam, V.B.; Yoon, Y.; Jung, J.; Hong, S.; Lee, H.; Eom, H.; Yeo, J.; et al. Sensitive wearable temperature sensor with seamless monolithic integration. Adv. Mater. 2020, 32, 1905527. [CrossRef] [PubMed]

14. Kim, J.Y.; Oh, J.Y.; Lee, T.I. Multi-dimensional nanocomposites for stretchable thermoelectric applications. Appl. Phys. Lett. 2019, 114, 043902. [CrossRef]

15. Suh, Y.D.; Jung, J.; Lee, H.; Yeo, J.; Hong, S.; Lee, P.; Lee, D.; Ko, S.H. Nanowire reinforced nanoparticle nanocomposite for highly flexible transparent electrodes: Borrowing ideas from macrocomposites in steel-wire reinforced concrete. J. Mater. Chem. C 2016, 5, 791-798. [CrossRef]

16. So, J.-H.; Dickey, M. Inherently aligned microfluidic electrodes composed of liquid metal. Lab Chip 2011, 11, 905-911. [CrossRef]

17. Park, Y.-L.; Chen, B.-R.; Wood, R.J. Design and fabrication of soft artificial skin using embedded microchannels and liquid conductors. IEEE Sens. J. 2012, 12, 2711-2718. [CrossRef]

18. So, J.-H.; Koo, H.-J.; Dickey, M.D.; Velev, O.D. Ionic current rectification in soft-matter diodes with liquid-metal electrodes. Adv. Funct. Mater. 2011, 22, 625-631. [CrossRef]

19. Parekh, D.; Ladd, C.; Panich, L.; Moussa, K.; Dickey, M.D. 3D printing of liquid metals as fugitive inks for fabrication of 3D microfluidic channels. Lab Chip 2016, 16, 1812-1820. [CrossRef]

20. Yang, Y.; Sun, N.; Wen, Z.; Cheng, P.; Zheng, H.; Shao, H.; Xia, Y.; Chen, C.; Lan, H.; Xie, X.; et al. Liquid-metal-based super-stretchable and structure-designable triboelectric nanogenerator for wearable electronics. ACS Nano 2018, 12, $2027-2034$. [CrossRef] [PubMed]

21. Zhang, R.; Ye, Z.; Gao, M.; Gao, C.; Zhang, X.; Li, L.; Gui, L. Liquid metal electrode-enabled flexible microdroplet sensor. Lab Chip 2020, 20, 496-504. [CrossRef]

22. Park, C.W.; Moon, Y.G.; Seong, H.; Jung, S.W.; Oh, J.-Y.; Na, B.S.; Park, N.-M.; Lee, S.S.; Im, S.G.; Koo, J.B. Photolithog-raphy-based patterning of liquid metal interconnects for monolithically integrated stretchable circuits. ACS Appl. Mater. Interfaces 2016, 8 , 15459-15465. [CrossRef] [PubMed]

23. Moon, Y.G.; Koo, J.B.; Park, N.-M.; Oh, J.-Y.; Na, B.S.; Lee, S.S.; Ahn, S.-D.; Park, C.W. Freely deformable liquid metal grids as stretchable and transparent electrodes. IEEE Trans. Electron Devices 2017, 64, 5157-5162. [CrossRef]

24. Wang, L.; Liu, J. Pressured liquid metal screen printing for rapid manufacture of high resolution electronic patterns. RSC Adv. 2015, 5, 57686-57691. [CrossRef]

25. Lazarus, N.; Bedair, S.S.; Kierzewski, I.M. Ultrafine pitch stencil printing of liquid metal alloys. ACS Appl. Mater. Interfaces 2017, 9 , 1178-1182. [CrossRef]

26. Park, T.H.; Kim, J.; Seo, S. Facile and rapid method for fabricating liquid metal electrodes with highly precise patterns via one-step coating. Adv. Funct. Mater. 2020, 30. [CrossRef] 
27. Dong, R.; Wang, L.; Hang, C.; Chen, Z.; Liu, X.; Zhong, L.; Qi, J.; Huang, Y.; Liu, S.; Wang, L.; et al. Printed stretchable liquid metal electrode arrays for in vivo neural recording. Small 2021, 17, 2006612. [CrossRef]

28. Kim, M.-G.; Brown, D.K.; Brand, O. Nanofabrication for all-soft and high-density electronic devices based on liquid metal. Nat. Commun. 2020, 11, 1-11. [CrossRef] [PubMed]

29. Tabatabai, A.; Fassler, A.; Usiak, C.; Majidi, C. Liquid-phase gallium-indium alloy electronics with microcontact printing. Langmuir 2013, 29, 6194-6200. [CrossRef]

30. Kim, J.H.; Seo, S. Fabrication of an imperceptible liquid metal electrode for triboelectric nanogenerator based on gallium alloys by contact printing. Appl. Surf. Sci. 2020, 509, 145353. [CrossRef]

31. Ma, Z.; Huang, Q.; Xu, Q.; Zhuang, Q.; Zhao, X.; Yang, Y.; Qiu, H.; Yang, Z.; Wang, C.; Chai, Y.; et al. Permeable superelastic liquid-metal fibre mat enables biocompatible and monolithic stretchable electronics. Nat. Mater. 2021, 20, 859-868. [CrossRef] [PubMed]

32. Nam, V.B.; Giang, T.T.; Koo, S.; Rho, J.; Lee, D. Laser digital patterning of conductive electrodes using metal oxide nano-materials. Nano Converg. 2020, 7, 23. [CrossRef]

33. Nam, V.B.; Shin, J.; Yoon, Y.; Giang, T.T.; Kwon, J.; Suh, Y.D.; Yeo, J.; Hong, S.; Ko, S.H.; Lee, D. Highly stable Ni-based flexible transparent conducting panels fabricated by laser digital patterning. Adv. Funct. Mater. 2019, 29. [CrossRef]

34. Kim, D.; Thissen, P.; Viner, G.; Lee, D.-W.; Choi, W.; Chabal, Y.J.; Lee, J.-B. Recovery of nonwetting characteristics by surface modification of gallium-based liquid metal droplets using hydrochloric acid vapor. ACS Appl. Mater. Interfaces 2012, 5, 179-185. [CrossRef]

35. Akbari, M.K.; Hai, Z.; Wei, Z.; Ramachandran, R.K.; Detavernier, C.; Patel, M.; Kim, J.; Verpoort, F.; Lu, H.; Zhuiykov, S. Sonochemical functionalization of the low-dimensional surface oxide of Galinstan for heterostructured optoelectronic applications. J. Mater. Chem. C 2019, 7, 5584-5595. [CrossRef]

36. Li, Y.; Tokizonno, T.; Liao, M.; Zhong, M.; Koide, Y.; Yamada, I.; Delaunay, J.-J. Efficient assembly of bridged $\beta$-Ga2O3 nan-owires for solar-blind photodetection. Adv. Funct. Mater. 2010, 20, 3972-3978. [CrossRef]

37. Hu, G.C.; Shan, C.X.; Zhang, N.; Jiang, M.M.; Wang, S.-P.; Shen, D.Z. High gain Ga_2O_3 solar-blind photodetectors realized via a carrier multiplication process. Opt. Express 2015, 23, 13554-13561. [CrossRef] [PubMed]

38. Kwon, Y.; Lee, G.; Oh, S.; Kim, J.; Pearton, S.J.; Ren, F. Tuning the thickness of exfoliated quasi-two-dimensional $\beta$-Ga2O3 flakes by plasma etching. Appl. Phys. Lett. 2017, 110, 131901. [CrossRef]

39. Xiao, P.; Gwak, H.-J.; Seo, S. Fabrication of a flexible photodetector based on a liquid eutectic gallium indium. Materials 2020, 13, 5210. [CrossRef]

40. Hwang, W.S.; Verma, A.; Peelaers, H.; Protasenko, V.; Rouvimov, S.; Xing, H.; Seabaugh, A.; Haensch, W.; Walle, C.V.; Galazka, Z.; et al. High-voltage field effect transistors with wide-bandgap $\beta$-Ga2O3 nanomembranes. Appl. Phys. Lett. 2014, 104, 203111. [CrossRef]

41. Boley, J.W.; White, E.L.; Kramer, R.K. Mechanically sintered gallium-indium nanoparticles. Adv. Mater. 2015, 27, 2355-2360. [CrossRef] [PubMed]

42. Lin, Y.; Cooper, C.; Wang, M.; Adams, J.J.; Genzer, J.; Dickey, M.D. Handwritten, soft circuit boards and antennas using liquid metal nanoparticles. Small 2015, 11, 6397-6403. [CrossRef] [PubMed]

43. Mingkuan, Z.; Siyuan, Y.; Wei, R.; Jing, L. Transformable soft liquid metal micro/nanomaterials. Mater. Sci. Eng. R Rep. 2019, $138,1-35$.

44. Lin, Y.; Liu, Y.; Genzer, J.; Dickey, M.D. Shape-transformable liquid metal nanoparticles in aqueous solution. Chem. Sci. 2017, 8, 3832-3837. [CrossRef] [PubMed]

45. Lu, Y.; Lin, Y.; Chen, Z.; Hu, Q.; Liu, Y.; Yu, S.; Gao, W.; Dickey, M.D.; Gu, Z. Enhanced endosomal escape by light-fueled liquid-metal transformer. Nano Lett. 2017, 17, 2138-2145. [CrossRef] [PubMed] 